\title{
HEART-VECTOR AND LEADS. PART III GEOMETRICAL REPRESENTATION
}

\author{
BY \\ H. C. BURGER AND J. B. VAN MILAAN \\ From the Physisch Laboratorium der Rijks Universiteit te Utrecht, Holland
}

Received May 31, 1948

In 1947 Wilson, Johnston, and Kossmann gave a 'generalization of the triangle-rule of Einthoven. In this paper they describe the use of a tetrahedron; their method is based on the same assumption as Einthoven's. We have : tried to give a rational description of the relation between heart-vector and leads, based on firmly established physical principles (Burger and van Milaan, 1947).

It is not self-evident that the electric action of the heart can be represented by one heart-vector. This is only correct if one is allowed to consider the dimensions of the heart as very small compared with those of the trunk. As a matter of course this can only be a useful approximation if the electrodes are applied far from the heart. In that case it does not matter very much whether the electric action originates in the one or in the other part of the heart muscle. As can be shown by mathematical analysis, the electric action of the heart can then be represented by one vector, acting in the "centre" of the heart. But for præcordial leads, i.e. electrodes near the heart, this simplification is certainly not entirely correct, and we shall return to this point at the end of this paper.

The formulation of the relation between heartvector and leads can be based on well-founded physical laws. It is then possible to say with certainty what is known $\dot{a}$ priori and what must be found by measurement. The lipear relation between

$$
L e a d=a X+b Y+c Z \text {. . . . . (1) }
$$

each lead and the rectangular components $X, Y, Z$ of the heart-vector is certain.

We have related these components to a "natural " co-ordinate-system, of which the $Z$-axis is parallel to the axis of the body and of which the $Y$-axis is perpendicular to the frontal plane (Burger and van Milaan, 1946, 1947).

As we have explained in the papers mentioned, the coefficients $a, b, c$, which are independent of the heart-vector, must be determined experimentally. For this one can use a phantom or measure on a cadaver. We have done the former ourselves (1.c.): the latter method is used by others (Fahr and Weber, 1915; de Waart and Storm, 1935; Storm, 1936; Wilson et al., 1947. But whichever method is used one must proceed objectively and leave the decision to the experiments. We have, therefore, provisionally accepted the values of the coefficients $a, b, c$, for a number of leads as the experiments gave them. By repeating the measurement and by varying the conditions we have tried to improve on their reliability.

With our phantom we have tried to reproduce the human body as closely as possible but are fully aware of the numerical imperfection of the result. We took as a basis our measurements of the electric conductivity of the living human body (Burger and van Milaan, 1943). In these measurements we have not noticed the influence of membranes that by their high resistance could determine the course of the electric current. Nor did preliminary measurements on (dead) membranes give a high value for this resistance either.

From various sides other systems are proposed instead of the Einthoven triangle, all of them rectangular. In these cases the electrodes are applied on the body in such a way, that the lines connecting them are mutually perpendicular and parallel to the axes of the above mentioned "natural" co-ordinate system (Schellong, 1939; Sulzer and Duchosal, 1945; Trocmé, 1946). As an argument.in its favour it is sometimes pat forward, that it is simpler, but it is not always realized that these systems are just as arbitrary as the equilateral triangle of Einthoven. Measurements on a phantom indicate that from a quantitative standpoint, these systems do not improve matters. In our opinion the lucky hit of Einthoven comes nearer to 
the true state of affairs than the rectangular systems mentioned above.

From some sides objections are raised against phantom measurements. Indeed we shall appreciate every suggestion for improvements on our phantom. Taking. into account the internal structure of the human body, this might be a rational way to approach the truth nearer and nearer as regards the numerical values of the coefficients $a, b, c$.

The attempts made by physicians to give the relation between heart-vector and leads have always led to geometrical representations (triangle rule, rectangular systems, equilateral tetrahedron). From these representations the analytical conclusions are drawn only in the second place and the result is given as a formula. This, however, is a nonphysical line of thought. The physical laws, governing the electric current density in the trunk as a consequence of the heart action, are of an analytical nature and this is also the case with their general consequence equation (1). One can proceed from this equation and it suffices in practice to find the heart-vector.

It cannot be denied, however, that a geometrical representation can be useful for the sake of an easy survey, but it has to be based on equation (1). Mathematically one has to derive from (1) the general geometrical representation, which is equivalent to this equation.

Those, who are acquainted with the vector calculus can deduce this representation from (1). The lead (=potential difference) is a scalar (undirected) quantity, i.e. a quantity characterized by only one single number, independent of the choice of the direction of the co-ordinate axes.* The quantities $X, Y, Z$ are vector components determining together the heart-vector. The value of each of these quantities depends on the choice of the direction of the axes. With another choice of the axes, therefore, we have three other components $X^{\prime}, Y^{\prime}, Z^{\prime}$. Their resultant, however, is the same heart-vector. Choosing other directions of the axes, the components $X, Y, Z$ will vary, while $a X+b Y+c Z$ must remain the same. Therefore, $a, b, c$ must vary too, i.e. depend on the direction of the axes. It can be proved that they are the components of a vector $\overrightarrow{(a, b, c)}$. The relation between this vector $\overrightarrow{(a, b, c)}$, the heart-vector $(\overrightarrow{X, Y, \vec{Z}})$ (or $\vec{H})$ and the scalar lead can be represented geometrically. The lead is the product of the projection of $\vec{H}$ on $(\overrightarrow{a, b, c)}$ and the length $\overrightarrow{(a, b, c)}$ of this last vector. $\varphi$ being the

* Other examples of scalar quantities are: temperature, mass, time. angle between the two vectors, this relation can be denoted in a more symmetrical form:

$$
\text { Lead }=\vec{H} \cdot(\overrightarrow{(a, b, c)} \cos \psi \ldots .
$$

This formula is analogous to the well-known relation:

$$
\text { work }=\text { force } \times \text { displacement } \times \cos \varphi
$$

The work is the scalar product of force and displacement just as in our case the lead is the scalar_product of the vector $\overrightarrow{(a, b, c)}$ and heart-vector $\vec{H}$.

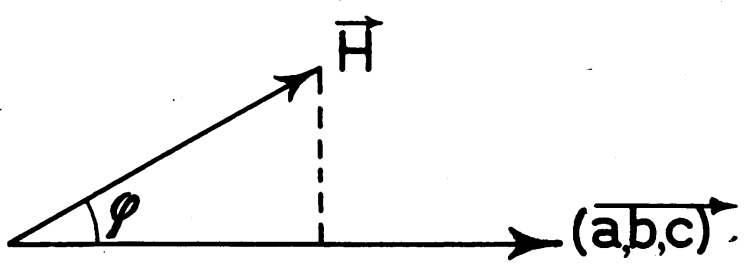

FIG. 1.-A lead is the product of the projection of the heart-vector $\vec{H}$ on the vector $\overrightarrow{(a, b, c)}$ and the length of the latter.

To determine $X, Y, Z$ (i.e. $\vec{H}$ ) we need three equations of the type (1) and, therefore, three leads (mutually independent). For simplicity we shall suppose that they have one electrode in common, for which as a rule the left leg $(F)$ is taken. If the potential differences between three electrodes and $F$ are known, we have three equations (1) to solve the three unknowns $X, Y, Z$. Interpreted geometrically, this means that there are three vectors

$\overrightarrow{(a, b, c)}$, originating in a common point, corresponding to $F$ (Fig. 2). The end points of these vectors correspond to the other three electrodes 1,2 , and 3, the position of which can be chosen arbitrarily. The points $F 123$ form a non-equilateral tetrahedron, the shape of which depends on the position of the electrodes on the body. The position of the tetrahedron, i.e. the direction of its edges with respect to the body is fixed, but it is obvious that its place may be chosen arbitrarily.

This tetrahedron makes it possible to represent geometrically the relation between heart-vector and leads. In order to determine lead (12), for example, the heart-vector must be projected on the edge (12) and the projection must be multiplied by the length of this edge (formula 2).

For a description of a general relation between heart-vector and leads a restriction to the three points $1,2,3$ is not allowed. With each point on the surface of our body corresponds a point in the space of the tetrahedron $F 123$. All those points, 4, 5, 6, together form a surface, on which are also situated the points $F 123$. An arbitrary point, say 10, can 


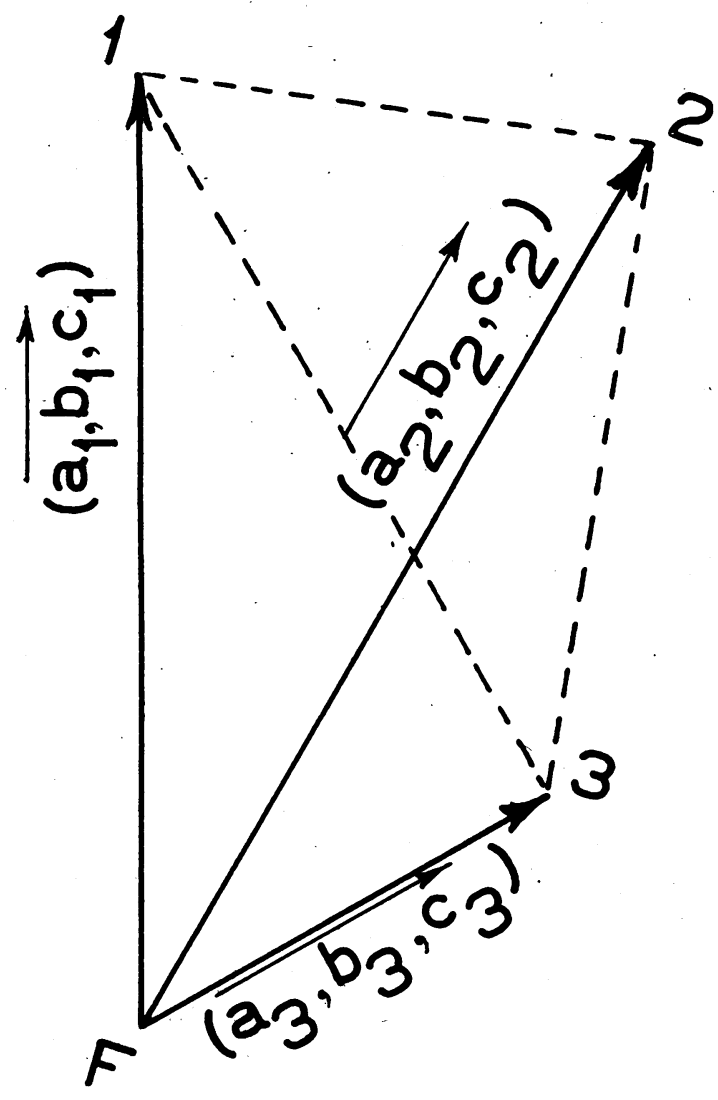

FIG. 2. - Three vectors $\overrightarrow{\left(a_{1} b_{1} c_{1}\right)} \overrightarrow{\left(a_{2} b_{2} c_{2}\right)}$ and $\overrightarrow{\left(a_{3} b_{3} c_{3}\right)}$ determining a tetrahedron with which the relation between the heart-vector and three leads can be described.

be determined with the aid of the phantom in the following way. In this phantom an electrode is applied in a position corresponding to the position of the electrode 10 on the body of the patient. On the phantom lead $10 F$, i.e. the potential difference of the electrodes 10 and $F$, arising from the known components $X, Y, Z$ of the vector of the artificial heart, is measured. The proportionality of actors being $a_{10}, b_{10}, c_{10}$, the lead is given by

$$
\text { Lead } 10 F=a_{10} X+b_{10} Y+c_{10} Z \text {. }
$$

In the space of the tetrahedron $F 123$ the vector $\overrightarrow{a_{10}, b_{10}, c_{10}}$ is drawn, the end-point of which has the co-ordinates $a_{10}, b_{10}, c_{10}$. This point is the "image" of the position of the electrode 10 on the surface of the body of the patient. The above mentioned surface, formed by all image-points (Fig. 3) can be used as follows, to find the relation between the heart-vector and any lead. Let $p$ and $q$ be two arbitrary positions of electrodes on the surface of the

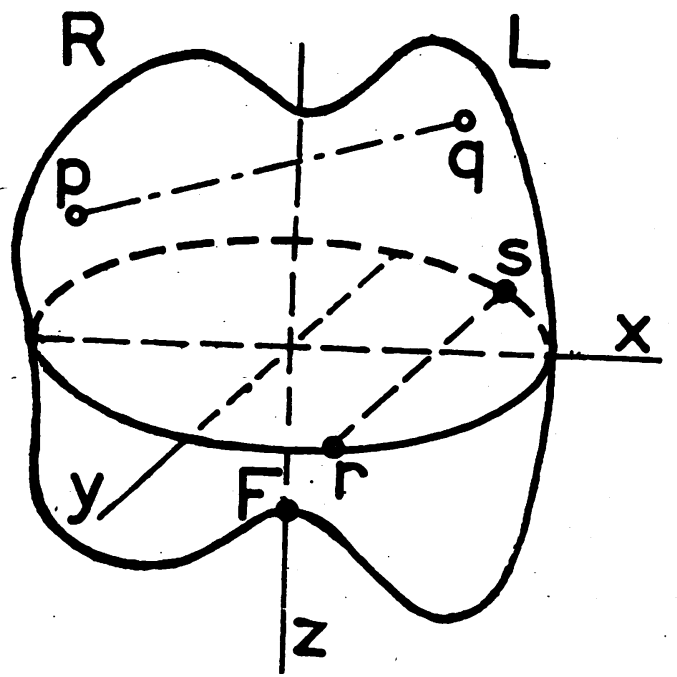

FIG. 3.-Surface in the image-space, corresponding to the surface of the human body. $X, Y, Z=$ co-ordinate axes, $r$ and $s=$ images of two electrodes, which give a potential difference, depending on the $Y$-component of the heart-vector only.

body. These give on the surface of Fig. 3 the "image-points" $p, q$. Now, lead $p q$ equals the product of the projection of the heart-vector, drawn in the correct direction in the image-space, on the line $p q$ and the length of this line (formula 2). It is possible to reverse this process and to deduce in a way, which we shall not explain here, the heart-vector from three leads, the image-surface of Fig. 3 being given.

An electrode within the body has also an imagepoint which can be found with the aid of the phantom. Such points are situated in the imagespace outside the surface of Fig. 3 so that, the nearer the electrode inside the human body is to the heart, the farther away they lie.

In a preceding paper (Burger and van Milaan, 1947) pairs of electrodes on the surface of the human body are mentioned, giving a potential difference that depends on one component of the heart-vector only. Then in equation (1) two coefficients are zero e.g. Lead=aX. The geometrical representation enables us to take a simple view of the problem. If a lead for example has to be found depending only on the postero-anterior component $Y$ of the heart-vector, the projections of the $X$ and $Z$ component on the line joining the imagepoints of the electrodes must be zero. This line, therefore, in the image must be parallel to the $Y$ axis. In Fig. 3, $r$ and $s$ are the images of electrodes, fulfilling this requirement. The relation between the position of the electrodes and the image-point 
keing known, the place where the electrodes must be applied is determined. It follows from the figure, that an infinite number of combinations $r s$ meet the requirement. It is possible to choose one electrode for instance arbitrarily. The other one $(s)$ is found as the point of intersection of the line $r s$ (parallel to the $Y$-axis) and the surface of the image: To use this method efficiently a practical combination must be chosen out of the infinite number of possibilities.

This geometrical representation can serve also for elucidating the meaning of the central terminal, introduced by Wilson (1935). This electrode is not applied on the surface of the body, but is the junction $C T$ (Fig. 4) of the ends of three equal resistances connected to the electrodes $R, L$, and $F$. If these resistances are high enough, it is easily proved, that the potential of $C T$ is the mean of the potentials of the electrodes $R, L$, and $F$. As it

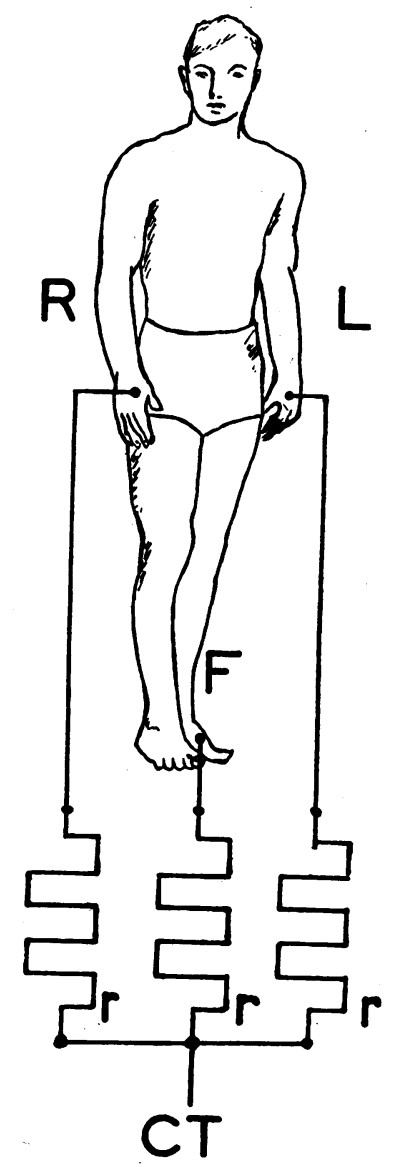

FIG. 4.-The central terminal $C T$ is the junction of the ends of three equal resistances $r$, connected to the electrodes $R, L$, and $F$. is not the potential itself that matters, but only the potential differences, it is formulated better by saying that the potential difference of $C T$ and an arbitrary electrode is the mean of the potential differences of $R, L$, and $F$ and this electrode.

It can be proved, that it is possible to indicate a point in the image-space (Fig. 3) corresponding to the central terminal. This image-point is the centre of gravity of triangle $R L F$. This point can be used for the geometrical determination of leads just as was explained above for points on the image-surface.

The image-point of the central terminal is situated inside that part of the image-space, that is enclosed by the image-surface. It is a point, that does not possess the special properties of the upper or lower side, the right or the left side of the imagesurface. Inside this it occupies a more or less central position, ignoring the fact that the surface image at the front of the plane $R L F$ (and of $C T$ ) has a much greater extension than at the back. The image has a pigeon breast.

Interpreted geometrically in this way, the central position of Wilson's central terminal seems to be explained satisfactorily. A more exact definition of a central point, in which not only the points $R L F$ are involved, but all the points of the surface of the body, is possible but seems to be hardly worth while.

It may be asked whether it is possible to obtain a point of mean potential with three electrodes other than $R L F$ in such a way, that this point has no potential difference with the central terminal. This can bé realized fairly approximately by experiment (personal communication by J. B. Kleyn). It can moreover be deduced from our geometrical considerations, that this can be obtained exactly and even in an infinite number of different ways. For there are an infinite number of triangles in the image-space, of which the angular points are situated on the image-surface and of which the centre of gravity coincides with the image-point of the central terminal. This, however, does not hold good for the image of the central terminal only, but for every point inside the image-surface.

In the preceding it has always been supposed, that the dimensions of the heart are very small compared with those of the trunk. In reality this is not the case so that the image-surface will depend on the position of that part of the heart muscle, of which the action is being studied. Comparing diametrically situated parts of the heart-muscle (e.g. heart point and heart base) this difference is appreciable as revealed by measurements on a phantom. But on the average the distances are much smaller so that in a first approximation this effect may be neglected. This, however, holds good only if the electrodes are applied at a rather large distance from 
the heart, as is the case for $R, L$, and $F$. A præcordial electrode, however, will be situated too near to the heart. The part of the heart muscle near the thoracic wall will contribute to the præcordial lead relatively more than the other parts. It is true that this reduces the value of the preceding considerations. But on the other hand it may enable one to find the place of disturbances in different parts of the heart muscle. This is understood quite well by physicians and it is for this very purpose that the præcordial leads are recommended. But the relation between the place of the defect and the electrocardiogram is determined in an empirical way only. A more correct comprehension of this relation is only to be obtained by a generalization of the exact physical treatment of the problem.

\section{SUMMARY}

The relation between heart-vector and leads can be represented geometrically. This representation is a generalization of Einthoven's triangle. It is possible to elucidate the meaning of the central terminal, proposed by Wilson.

\section{REFERENCES}

Burger, H. C., and van Milaan, J. B. (1943). Acta med. Sulzer, R., and Duchosal, P. W. (1945). Cardiologia, IX, scand., 114, 584.

- - - (1946). Brit. Heart J., 8, 157.

- - (1947). Ibid., 9, 154.

Fahr, G., and Weber, A. (1915). Dtsch. Arch. Klin. Med., 117.

Schellong, F. (1939). Grundzüge einer klinischen Vektordiagraphie des Herzen, Berlin, Springer.

Storm, C. J., (1936). Thesis, Batavia.
Fasc. 1/2, 106.

Trocmé, Ch. (1946). Arch. Mal. Coeur, 39, 80.

de Waart, A., and Storm, C. J., (1935). Arch. Néerl. Physiol. 20, 255.

Wilson, F. N., Johnston, F. D., Macleod, A. G., and Barker, P.' S. (1935). Amer. Heart J. 9, 447.

,,-- and Kossmann, C. F. (1947). Ibid., 33, 594. 